\title{
Análise temporal da fragmentação florestal no leste da Amazônia legal
}

\section{Fragmentation temporal analysis of forest at eastern legal Amazon}

Leila Sheila Lisboa - Doutora em Ciências, na área de concentração Engenharia de Sistemas Agrícolas, pela Escola Superior de Agricultura "Luiz de Queiroz" (ESALQUSP). Pesquisadora bolsista do Campus de Pesquisa do Museu Paraense Emílio Goeldi. E-mail: lisboa.leilass@gmail.com.

Arlete Silva de Almeida - Doutora em Ciências Ambientais, pela Universidade Federal do Pará (UFPA). Tecnóloga do Ministério de Ciências e Tecnologia lotada no Museu Paraense Emílio Goeldi. E-mail: arlete@museu-goeldi.br.

Wanja Janayna Lameira - Doutora em Ciências Ambientais, pela Universidade Federal do Pará (UFPA). Pesquisadora bolsista no Museu Paraense Emílio Goeldi. E-mail: wjlameira@hotmail.com.

\section{Resumo}

Neste estudo foram avaliadas as mudanças estruturais dos fragmentos florestais superiores a $1 \mathrm{~km}^{2}$ localizados na porção norte da área do Centro de Endemismo Belém (CEB). Os dados foram obtidos de imagens orbitais do satélite Landsat 5 e 8 , dos anos de 1985 e 2018, e se aplicaram sete índices de análise da paisagem no sistema computacional FRAGSTATS v. 4.2. Foram considerados tamanho, forma e proximidade dos fragmentos. O resultado da análise temporal evidenciou que, nesse período, houve um aumento do número (quantidade) de fragmentos, redução do tamanho médio da área total dos mesmos, além da redução significativa da agregação, promovendo o aumento no nível de fragmentação que pode afetar a manutenção da biodiversidade local, pois se refere à diminuição da quantidade de área total de habitat na paisagem da área de estudo.

\section{Palavras-chave}

Centro de Endemismo Belém. Fragmentos Florestais. Amazônia. Geotecnologias.

\begin{abstract}
In this study the structural changes of forest fragments greater than $1 \mathrm{~km}^{2}$ located in the northern portion of the Belém Endemism Center (CEB) area were evaluated. Data were obtained from orbital images of the Landsat 5 and 8 satellite from 1985 and 2018 and seven landscape analysis indices were applied to the FRAGSTATS v. 4.2. Size, shape and proximity of the fragments were considered. The result of the temporal analysis showed that, during this period, there was an increase in the number (quantity) of fragments, reduction in the average size of the total area of the fragments, and a significant reduction of aggregation promoting the increase in the fragmentation level that may affect the fragmentation maintenance of local biodiversity, as it refers to the decrease in the amount of total habitat area in the study area landscape.
\end{abstract}

\section{Keywords}

Center of Endemism Belém. Forest Fragments. Amazon. Geotechnologies. 


\section{INTRODUÇÃO}

A capacidade humana de apropriação da natureza, para seu conforto, tem alterado as dinâmicas socioambientais das paisagens, modificando-as num complexo de unidades interativas, ocasionando, em sua maioria, manchas isoladas do habitat natural, com impactos negativos, visto que reduz o tamanho das populações, favorece o acasalamento entre indivíduos aparentados (endocruzamento) e a baixa variabilidade genética (ANDREN, 1994; PÉRICO; CEMIN, 2006). Além do fato de que existem limitações biofísicas, causando mudanças nas relações ecológicas entre a fauna, a flora e o meio abiótico (RIBEIRO; MARQUES, 2005), já que as interações dos organismos entre si e com o meio físico ou abiótico se estabelecem de modo a permitir a reprodução das populações e a manutenção da diversidade biológica do ecossistema, essencial para a sua autorregulação e perpetuação (VARJABEDIAN; MECHI, 2013).

Estudos têm abordado esta problemática (SOARES, 2004; PIROVANI; GOMES DA SILVA; ROSA DOS SANTOS, 2015) e, diante disto, a adoção de estratégias de mitigação em áreas antrópicas pode subsidiar novas alternativas de planejamento sustentável, a partir do conhecimento sobre a fragmentação de habitats e a supressão da vegetação nativa, consideradas as maiores ameaças à biodiversidade global (DA CRUZ SILVA et al., 2013; MYERS et al., 2000). Neste contexto, a devastação dos habitats naturais, particularmente das florestas, é considerada fator determinante na extinção da biodiversidade (ALBAGLI, 2010).

Na Amazônia Legal, tal fragmentação está relacionada com derrubada, queima e posterior substituição da floresta nativa para a atividade agropecuária. $\mathrm{O}$ uso do solo no entorno dos fragmentos favorece reações em cadeia que avançam além do limite da borda para dentro do fragmento (TABARELLI; LOPES; PERES, 2008).

Têm-se como principais consequências da fragmentação das paisagens a perda de habitat, a alteração do tamanho, quantidade e isolamento dos fragmentos, as mudanças nas condições ambientais entre as bordas das manchas e o seu interior (MALCOLM, 1994; LAURANCE; GASCON, 1997; FAHRIG, 2003; MCGARIGAL, 2002). Em síntese, quanto mais fragmentada estiver a área, maior será a heterogeneidade da paisagem, o que implica no aumento das perturbações nesses ambientes (DA SILVA; MELO, 2014).

A capacidade de quantificar a estrutura da paisagem é um pré-requisito para a compreensão das intervenções antrópicas (HEROLD; COUCLELIS; CLARKE, 2005). Neste estudo, utilizou-se uma série de métricas para entender as relações entre os padrões espaciais e os processos ecológicos do leste da 
Amazônia Legal, possibilitando sua quantificação, mensuração e entendimento da sua estrutura e processo de fragmentação na paisagem. A vantagem desse método é a alta capacidade de caracterizar, comparar, identificar diferenças e determinar relações entre os processos funcionais e os padrões das paisagens (CABACINHA; CASTRO; GONÇALVES, 2010), além de demonstrar de maneira rápida as transformações ambientais ocorridas (UUEMAA; MANDER; MARJA, 2013).

Essas métricas são conhecidas como métricas da paisagem e são índices quantitativos que descrevem aspectos composicionais e espaciais da paisagem (LEITÃO; AHERN, 2002) baseados, exclusivamente, em mapas categóricos, provenientes de imagens de sensoriamento remoto (MCGARIGAL, 2002; KUPFER, 2012). Portanto, tais métricas funcionam como suporte científico tanto da Geografia como da Ecologia de Paisagens, nos estudos de gestão territorial e a conservação biológica (METZGER, 2001). Associado a isto, observa-se também o avanço do emprego das geotecnologias, que, em conjunto, contribuíram consideravelmente nos estudos de Paisagem mediante o uso de imagens de satélite, cujas principais propriedades são a repetitividade, definição (resolução espacial), múltiplas bandas espectrais, que têm possibilitado a detecção de alterações na cobertura vegetal, os diferentes tipos de uso da terra, caracterização e quantificação de tais alterações, em especial na região amazônica.

Assim, o objetivo deste estudo foi mapear, quantificar e analisar as mudanças estruturais dos fragmentos florestais superiores a $1 \mathrm{~km}^{2}$ localizados na parte norte da área do Centro de Endemismo Belém (CEB), para os anos de 1985 e 2018. Esta região tem protagonismo na Amazônia Oriental por se tratar de uma área de ocupação mais antiga das frentes pioneiras (ALMEIDA; VIEIRA, 2010). $\mathrm{O}$ ano de 1985 corresponde à data mais antiga, enquanto que 2018 representa a data mais recente, ambas disponíveis no catálogo de imagens Landsat da região.

\section{MATERIAL E MÉTODOS}

\section{1 ÁREA DE ESTUDO}

O estudo da fragmentação florestal foi realizado no quadrante localizado entre os paralelos $0^{\circ} 31^{\prime} 35,8^{\prime \prime}$ e $3^{\circ} 49^{\prime} 29,4^{\prime \prime}$ de latitude sul e entre os meridianos $44^{\circ} 20^{\prime} 52,5^{\prime \prime}$ e 49 40'57,6" de longitude oeste, que correspondem à parte norte do Cento de Endemismo Belém (Figura 1). Este recorte espacial chama atenção pela intensa ocupação antrópica e a existência de um grande número de fragmentos de florestas nativas. 
Trata-se de uma área com aproximadamente 154.819,25 km², localizada a leste da Amazônia Legal, que contempla parte dos estados do Pará e Maranhão e contempla Unidades de Conservação e outros grandes fragmentos florestais, indicando grande relevância ambiental na região.

Figura 1 - Mapa da delimitação da área de estudo

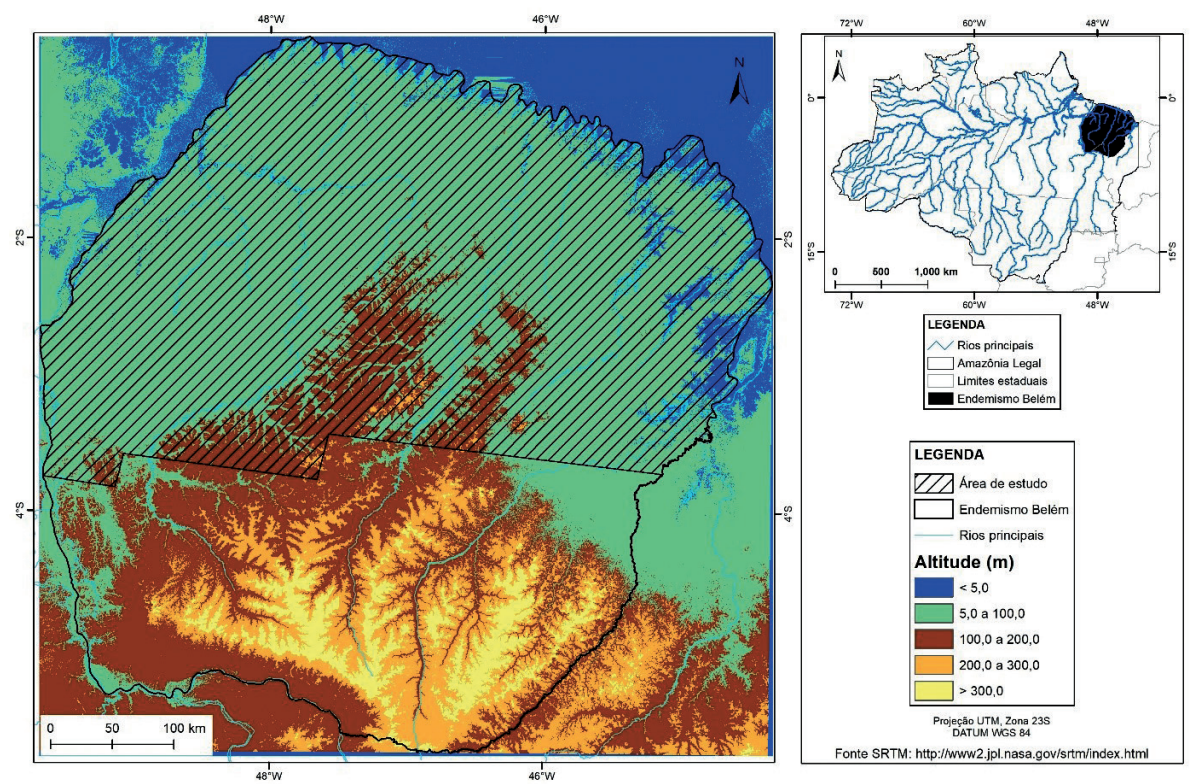

Elaboração: Autoras, 2018.

\subsection{PROCEDIMENTOS METODOLÓGICOS}

\subsubsection{Análise multitemporal de floresta nativa}

Para a identificação e evolução do grau de perda dos fragmentos florestais, foram utilizadas as imagens derivadas de produtos de sensoriamento remoto, que são fontes de produção de mapas de diversas temáticas (uso e cobertura da terra, desmatamento, floresta nativa, focos de incêndio, entre outras), além de importantes fontes de dados em estudos que envolvem áreas florestadas na Amazônia Legal. Portanto, foram usadas nove imagens de satélite da série Landsat 5 do ano de 1985 e nove imagens Landsat 8 do ano de 2018, que se encontram nas seguintes órbitas-ponto: 221-61; 221-62; 222-61; 222-62; 223-60; 223-61; 223-62; 224-61; 224-62 (Figura 2). Cada sistema de referência (órbitaponto) cobre uma área de 185 x $185 \mathrm{~km}^{2}$, adquiridas gratuitamente no site Glovis (HOUSKA; JOHNSON, 2012). 
Figura 2 - Imagens Landsat 8 do ano de 2018 da área de Endemismo Belém com suas respectivas órbitas-ponto

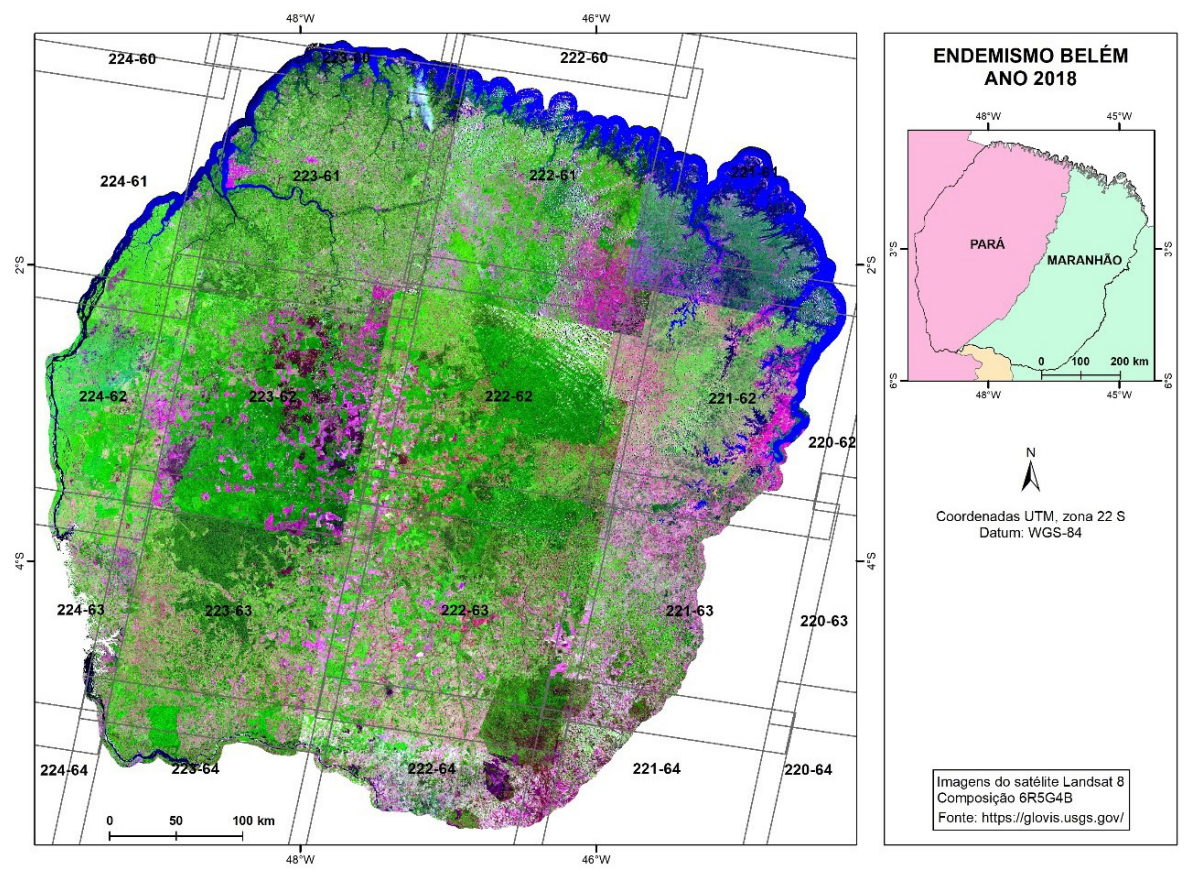

Elaboração: Autoras, 2018.

Esta seleção foi baseada em imagens considerando o mínimo de cobertura de nuvens e datas mais próximas possíveis das primeiras aquisições da série Landsat, liberadas gratuitamente, além de imagens mais recentes, o que possibilitou uma análise espaço-temporal da paisagem estudada. As aquisições mais antigas (desde 1972), disponíveis gratuitamente, possibilitam a realização do monitoramento de fenômenos naturais e antrópicos sobre uma série temporal superior a 40 anos.

Em seguida, organizaram-se as imagens em um único banco de dados para a geração de uma área comum a todas as órbitas-ponto. Sendo assim, a área estudada é a intersecção dos quatro anos classificados. O que não pertencia a este critério não foi analisado.

Este procedimento foi gerado a partir de técnicas de processamento digital de imagens- PDI, utilizadas para otimizar o trabalho de mapeamento para diversos fins, gerando grande quantidade de informações sobre a área de estudo, mesmo antes dos trabalhos de campo (DE PAULA MIRANDA et al., 2016). Os procedimentos foram realizados no programa Emvironment for Visualizing 
Images (ENVI), v.4.5: junção das bandas multiespectrais (1-5 e 7) para o Landsat 5 e bandas multiespectrais (1-7 e 9) para o Landsat 8; pré-processamento das imagens; classificação das imagens, com a utilização do classificador ISO-DATA, pois não havia pontos obtidos em campo que representassem toda a área de estudo; aplicação da matemática de bandas para transformar os dados de inteiro para bytes; interpretação visual das imagens Landsat 5 e 8 . Nesta etapa, nas imagens Landsat 5, foi realizada uma composição colorida (5R4G3B) e, nas imagens Landsat 8, foi usada a composição colorida (6R5G4B) ambas na escala de 1:80.000. Os critérios usados na identificação e determinação desta classe temática foram forma, relacionada à geometria do objeto; tamanho, que varia conforme a resolução espacial da imagem; tonalidade, relacionada à quantidade de energia refletida por um objeto; e textura, que pode ser lisa ou rugosa.

Ao final da primeira classificação (1985), foi gerado um arquivo rasterizado apenas com manchas florestais de 1985, que serviu de base para a classificação dos fragmentos que ainda persistiam no ano de 2018.

Após a classificação temática das áreas de floresta nativa para cada ano analisado, foi criado um banco de dados espaciais usando a ferramenta de Geoprocessamento, evidenciado pelos Sistemas de Informações Geográficas (SIG), que são plataformas de banco de dados para a edição e manipulação de dados espaciais. Os SIG permitem a obtenção qualitativa e quantitativa de dados computacionais geográficos possibilitando a gestão dos recursos e aplicação de técnicas otimizadas baseadas em informações georreferenciadas. Esta etapa permitiu a análise temporal entre os anos de 1985 e 2018.

Posteriormente, as áreas de fragmentos florestais foram identificadas de acordo com o tamanho, selecionando-se apenas os fragmentos superiores a $1 \mathrm{~km}^{2}$, por meio da tabela de atributos. Por fim, as classificações foram reclassificadas e convertidas para .tif, para sua inserção posterior no programa de métricas da paisagem, mantendo a resolução espacial de $900 \mathrm{~m}^{2}$.

\subsubsection{Fragmentação da paisagem}

Para a caracterização da composição e configuração da paisagem incluindo a quantificação de mudanças espaço-temporais, foi desenvolvido um grande número de métricas para quantificar as manchas quanto a sua forma, tamanho, isolamento e o mosaico da paisagem em função de sua riqueza, diversidade e conectividade (MCGARIGAL; MARKS, 1995; MCGARIGAL; CUSHMAN, 2002). Essas métricas são conhecidas como métricas da paisagem e são índices quantitativos que descrevem aspectos composicionais 
e espaciais da paisagem (LEITÃO; AHERN, 2002) baseados, exclusivamente, em mapas categóricos, provenientes de imagens de sensoriamento remoto (MCGARIGAL, 2002; KUPFER, 2012).

As métricas foram computadas no programa Spatial Pattern Analysis (FRAGSTATS) (MCGARIGAL; MARKS, 1995). O programa Fragstats de estatística espacial é considerado padrão entre as ferramentas computacionais atualmente disponíveis para a análise quantitativa da estrutura da paisagem (LANG; BLASCHKE, 2009). Aceita imagens raster numa variedade de formatos: ArcGrid, ASCII, IDRISI, entre outros. Aceita uma imagem classificada, realiza pesquisas na imagem para buscar fragmentos contíguos de cada tipo e, então, calcula um conjunto compreensivo de valores de paisagem e índices baseados nos fragmentos encontrados.

Alguns arquivos são opcionais, porém necessários, para simular as métricas com mais facilidade. Neles, as classes são configuradas como área de background ou área de borda. Neste trabalho, foi usada somente a tabela de descritores de classes (Tabela 1). Esta tabela permite a especificação da descrição para cada valor numérico das classes. Nela são especificadas as classes em que os cálculos são realizados, permitindo a exclusão de determinadas classes. Assim, exceto a classe de floresta nativa, todas as outras foram consideradas como background, com o objetivo de calcular apenas as métricas nos fragmentos florestais.

Tabela 1 - Tabela de descritores das classes de interesse

ID, Name, Enabled,

Is Background

$1, \mathrm{FN}$, true, false

2, OUTROS, false, true

Elaboração: Autoras, 2018.

Foram utilizadas nove métricas da paisagem, relacionadas a tamanho, forma e proximidade dos fragmentos. Por meio das métricas de tamanho, obtiveram-se a área de cada fragmento, a área total da classe dos fragmentos, a porcentagem da área da classe em relação a área total da paisagem analisada e o tamanho médio dos fragmentos, descritos a seguir:

a) NP (Número de fragmentos): Grande número de fragmentos geralmente é equiparado à riqueza estrutural (LANG; BLASCHKE, 2009). Maiores valores de NP indicam maior fragmentação, e menores valores indicam união ou extinção 
de fragmentos de mesma classe (MCGARIGAL, 2002; SOUZA et al., 2014). NP deve ser analisado juntamente com a métrica CA e PLAND. Por exemplo: Se NP diminui, porém CA e PLAND aumentam, isso indica que houve união de fragmentos, mas se NP diminui, entretanto CA e PLAND também diminuem, indica que fragmentos foram extintos da paisagem (MCGARIGAL, 2002). Seu cálculo é realizado de acordo com a equação 1:

$$
\mathrm{NP}=n_{\mathrm{i}}
$$

onde $n_{\mathrm{i}}$ número de manchas em uma paisagem de uma determinada classe $i$.

b) CA (Área total dos fragmentos): É um índice de composição da paisagem. Corresponde a área de todos os fragmentos da classe (MCGARIGAL; MARKS, 1995). Seu cálculo é realizado de acordo com a equação 2:

$$
C A=\sum_{j=1}^{n} a_{i j} \times \frac{1}{10000}
$$

onde $\mathrm{a}_{i j}$ área $\left(\mathrm{m}^{2}\right)$ do fragmento $i j$.

c) PLAND (Percentual de fragmentos de mesma classe): De forma geral, fragmentos pequenos têm uma menor variedade de habitats do que os fragmentos grandes, o que também acarreta uma perda de espécies (LAURANCE; VASCONCELOS, 2009). Seu cálculo é realizado de acordo com a equação 3:

$$
P L A N D=P_{i}=\frac{\sum_{j=1}^{n} a_{i j}}{A} \times 100
$$

onde $\mathrm{P}_{i}=$ proporção da paisagem ocupada pela mancha $i$;

$\mathrm{a}_{i j}=$ área $\left(\mathrm{m}^{2}\right)$ da mancha $i j$;

$A=$ área total da paisagem $\left(\mathrm{m}^{2}\right)$.

d) AREA (Área do fragmento): Quanto menor o fragmento, maior a influência dos fatores externos sobre ele, causados pela intensidade do efeito de borda, pois os fragmentos ficam mais vulneráveis às mudanças físicas do ambiente (COLLI et al., 2003). Seu cálculo é realizado de acordo com a equação 4:

$$
A R E A=a_{i j} \times \frac{1}{10000}
$$

onde $\mathrm{a}_{i j}=$ área $\left(\mathrm{m}^{2}\right)$ da mancha $i j$.

e) AREA-MN (Tamanho médio dos fragmentos) e PD (Densidade dos fragmentos): A união desses dois índices está diretamente relacionada à qualidade 
do habitat, pois quanto maior o tamanho dos fragmentos e quanto mais próximos uns dos outros, maior a persistência e estabilidade dos processos ecológicos atuantes na paisagem (REMPEL, 2009). Seu cálculo é realizado de acordo com a equação 5:

$$
A R E A_{M N}=\frac{\sum_{j=1}^{n} x_{i j}}{n_{i}}
$$

onde $\mathrm{x}_{i j}=$ mancha de uma determinada classe $\mathrm{i}$.

f) LPI (Índice do maior fragmento na paisagem): Determina a porcentagem que o maior fragmento ocupa na paisagem. A variação deste índice avalia a redução da diversidade biológica (POLETTO; METZGER, 2002). Maior valor favorece dispersores, polinizadores e dispersão de propágulos, abastecendo fragmentos menores (SOUZA et al., 2014). Seu cálculo é realizado de acordo com a equação 6:

$$
L P I=\frac{\max \left(a_{i j}\right)_{j=1}^{n}}{A} \times 100
$$

onde $\mathrm{a}_{i j}=$ área $\left(\mathrm{m}^{2}\right)$ de uma mancha $i j$; $A=$ área total da paisagem $\left(\mathrm{m}^{2}\right)$.

g) TE (Total de bordas): É uma medida absoluta do comprimento total. O total de bordas geralmente é equiparado com riqueza de estrutura; também pode significar fragmentação (LANG; BLASCHKE, 2009); quando a floresta é fragmentada a quantidade de bordas aumenta dramaticamente (LAURENCE; VASCONCELOS, 2009). A qualidade dos remanescentes também se altera, trazendo prejuízos adicionais às populações florestais (ANDRÉN, 1994; TURNER et al., 2001). Seu cálculo é realizado de acordo com a equação 8:

$$
T E=\sum_{k=1}^{m} e_{i k}
$$

onde $\mathrm{e}_{i k}=$ comprimento total de borda em uma paisagem com determinada classe $i$.

h) AI (Índice de agregação): Valores próximos de 100\% indicam maior agregação e, portanto, menos isolamento dos fragmentos da classe (CABACINHA; CASTRO; GONÇALVES, 2010). Também significa que grandes e contínuas manchas são encontradas na paisagem (PEREIRA et al., 2001). Seu cálculo é realizado de acordo com a equação 9:

$$
A I=\left\lfloor\frac{g_{i i}}{\max \rightarrow g_{i i}}\right\rfloor \times 1000
$$

onde $\mathrm{g}_{i i}=$ número de ligações entre pixels de uma determinada classe $i$ baseada no método de contagem simples. 


\section{RESULTADOS E DISCUSSÃO}

\subsection{ANÁLISE ESPAÇO-TEMPORAL DE FLORESTA NATIVA}

Os resultados das classificações dos anos de 1985 e 2018 demonstram que, neste período de 33 anos, houve alterações significativas na cobertura de floresta nativa, pois em 1985 a área total de floresta nativa era de 8.063.296,0 ha e, em 2018, a área total foi reduzida para 4.564.078,0 ha, ou seja, metade da área de floresta nativa foi convertida para outras classes. A principal modificação observada foi na parte oeste da área de estudo, ou seja, a maioria de fragmentos convertidos em determinados tipos de usos ou outro tipo de cobertura florestal foi no estado do Pará, talvez por ser também o estado com os maiores fragmentos florestais em 1985 (Figura 3). Sabe-se que, neste período, têmse datas marcadas por altas taxas de desmatamento, o que culminou com o maior índice de desmatamento na Amazônia, em 2004, apontado pelo Instituto Nacional de Pesquisas Espaciais (INPE).

Figura 3 - Fragmentos florestais preservados e convertidos entre os anos de 1985 e 2018

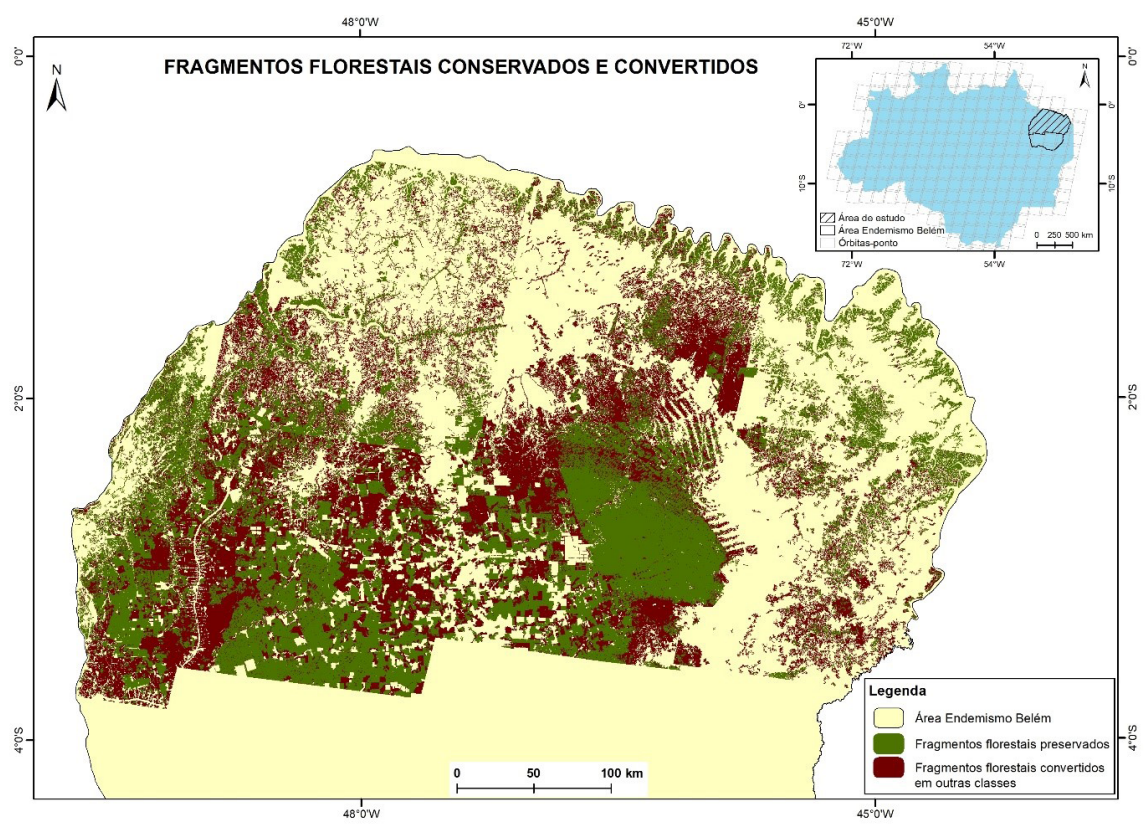

Elaboração: Autoras, 2018. 
2.1.1 Análise dos fragmentos florestais da paisagem para os anos de 1985 e 2018

O número de fragmentos de floresta nativa (NP) mensurados foi de 1.738,0 em 1985, e 2.480,0 no ano de 2018, indicando um aumento no NP, acompanhado de uma redução em área passando de 8.063.296,0 ha para 4.564.078,0 ha, além da redução de PLAND passando de 49\% para 28\%. Isto evidenciou o aumento da fragmentação durante este período (MCGARIGAL, 2002; SOUZA et al., 2014), a redução da composição da paisagem (MCGARIGAL; MARKS, 1995) e de forma geral, a redução da variedade de habitats, acarretando perda de espécies (LAURANCE; VASCONCELOS, 2009).

O índice AREA_MN indica redução progressiva no tamanho dos fragmentos florestais. O tamanho médio destes fragmentos foi de 4.639.411,0 ha (1985) para 1.840.354 ha (2018) indicando um aumento no grau de fragmentação.

Tabela 2 - Descrição dos parâmetros métricos dos fragmentos florestais utilizados e gerados pelo FRAGSTATS para os anos de 1985 e 2018

\begin{tabular}{l|l|r|r}
\hline \multicolumn{2}{c}{\begin{tabular}{c} 
Parâmetros \\
\cline { 3 - 4 } \multicolumn{2}{c|}{1985}
\end{tabular}} & \multicolumn{2}{c}{2018} \\
\hline NP & Número de fragmentos & $1.738,0$ & $2.480,0$ \\
\hline CA & Área da classe fragmentos & 8.063 .296 & 4.564 .078 \\
\hline PLAND & \% de floresta & 49,0 & 28,0 \\
\hline AREA_MN & Área média dos fragmentos & $4639.411,0$ & $1840.354,0$ \\
\hline LPI & Índice do maior fragmento & 72.6 & 5.07 \\
\hline TE & Total de bordas & 43.906 .110 & 35.658 .330 \\
\hline AI & Agregação & 96,7 & 94,0 \\
\hline
\end{tabular}

NP: Número de fragmentos; CA: Área total dos fragmentos; PLAND: Percentual de fragmentos de mesma classe; AREA_MN: Tamanho médio dos fragmentos; LPI: Índice do maior fragmento na paisagem; TE: Total de bordas; AI: Índice de agregação.

Fonte: Autoras (2018).

A porcentagem ocupada pelo maior fragmento da paisagem (LPI) era de aproximadamente, $73 \%$ (1985), chegando a 5\% no ano de 2018, o que indica a redução da diversidade biológica (POLETTO; METZGER, 2002) no maior fragmento florestal mapeado em 1985.

Houve aumento do número de bordas (TE) na área estudada. Em 1985 havia 43.906.110,0 m de borda para cada hectare da paisagem, valor que foi reduzido para 35.658.330,0 $\mathrm{m}$ em 2018, indicando que a qualidade dos remanescentes está sendo alterada, trazendo prejuízos adicionais às populações florestais (ANDREN, 1994; TURNER et al., 2001). 
A agregação entre os fragmentos de floresta nativa passou de 97\% (1985) para 94\% (2018), evidenciando que ainda existem grandes e contínuas manchas na paisagem estudada (PEREIRA et al., 2001).

\section{CONSIDERAÇÕES FINAIS}

Os fragmentos florestais do Centro de Endemismo Belém, para os anos de 1985 e 2018, estão aumentando em relação ao número (quantidade) e diminuindo em tamanho e agregação dos mesmos. Esta fragmentação de habitat está relacionada ao avanço das atividades agropecuárias e do processo de urbanização na Amazônia Oriental, considerados atualmente as principais forçantes; consequentemente, pode pôr em risco a manutenção da biodiversidade local. Estes dados chamam atenção, pois esta é a realidade vivenciada pela maioria das florestas tropicais no mundo, em especial da floresta amazônica.

O presente trabalho ressalta a importância que avaliações sobre a análise temporal dos habitats naturais, particularmente das florestas, são determinantes em estudos relacionados à biodiversidade, pois permite subsidiar a adoção de estratégias de mitigação em áreas antrópicas com novas alternativas de planejamento sustentável, a partir do conhecimento sobre a fragmentação de habitats e a supressão da vegetação nativa, consideradas as maiores ameaças à biodiversidade global.

\section{REFERÊNCIAS}

ALBAGLI, S. Amazônia: fronteira geopolítica da biodiversidade. Parcerias estratégicas, Brasília, v. 6, n. 12, p. 05-19, 2010.

ALMEIDA, A. S.; VIEIRA, I. C. G. Centro de Endemismo Belém: status da vegetação remanescente e desafios para a conservação biológica e restauração ecológica. Revista de Estudos Universitários, Sorocaba, v. 36, p. 95-111, 2010.

ANDRÉN, H. Effects of habitat fragmentation on birds and mammals in landscapes with different proportions of suitable habitat: a review. Oikos, v. 71, n. 3, p. 355-366, 1994.

CABACINHA, C. D.; CASTRO, S. S.; GONÇALVES, D. A. Análise da estrutura da paisagem da alta bacia do Rio Araguaia na savana brasileira. Floresta, Curitiba, v. 40, n. 4, p. 675-690, out./dez. 2010. 
COLLI, G. R. et al. A fragmentação dos ecossistemas e a biodiversidade brasileira: uma síntese. In: RAMBALDI, D. M.; OLIVEIRA, D. A. S. (org.). Fragmentação de ecossistemas: causas, efeitos sobre a biodiversidade e recomendações de políticas públicas. Brasília: MMA/SBF, 2003. p. 317-326.

DA CRUZ SILVA, A. C. et al. Aspectos de ecologia de paisagem e ameaças à biodiversidade em uma unidade de conservação na Caatinga, em Sergipe. Revista Árvore, v. 37, n. 3, p. 479-490, 2013.

DA SILVA, M. S. F.; MELO, R. Padrões espaciais de fragmentação florestal na Flona do Ibura-Sergipe (spatial patterns of forest fragmentation in the Flona Ibura-Sergipe). Revista Mercator, Fortaleza, v. 13, n. 3, p. 121-137, 2014.

DE PAULA MIRANDA, M. et al. Emprego de Técnicas de Processamento Digital em Imagens Landsat 8 para Mapeamento Geológico. Geologia, v. 29, n. $1,2016$.

FAHRIG, L. Effects of habitat fragmentation on biodiversity. Annual Review of Ecology, Evolution, and Systematics, Palo Alto, v. 34, p. 487-515, 2003.

HEROLD, M.; COUCLELIS, H.; CLARKE, K. C. The role of spatial metrics in the analysis and modeling of urban land use change. Computers, environment and urban systems, v. 29, n. 4, p. 369-399, 2005.

HOUSKA, T. R.; JOHNSON, A. P. GloVis. Washington: U.S. Geological Survey General, 2012. 2 p. (General Information Product, 137).

KUPFER, J.A. Landscape ecology and biogeography: rethinking landscape metrics in a post-FRAGSTATS landscape. Progress in Physical Geography, London, v. 39, n. 3, p. 388-404, 2012.

LANG, S.; BLASCHKE, T. Análise da paisagem com SIG. São Paulo: Oficina de Textos, 2009. 424 p.

LAURANCE, W. F.; GASCON, C. How to creatively fragment a landscape. Conservation Biology, New York, v. 11, n. 2, p. 577-579, 1997.

LAURANCE, W. F; VASCONCELOS, H. L. Conseqüências ecológicas da fragmentação florestal na Amazônia. Oecologia Brasiliensis, Rio de Janeiro, v. 13, n. 3, p. 434-451, 2009.

LEITÃO, A. B.; AHERN, J. Applying landscape ecological concepts and metrics in sustainable landscape planning. Landscape and Urban Planning, Amsterdam, v. 59, n. 2, p. 65-93, 2002. 
MALCOLM, J. R. Edge effects in central Amazonian forest fragments. Ecology, New York, v. 75, n. 8, p. 2438-2445, 1994.

MCGARIGAL, K. Landscape pattern metrics. In: EL-SHAARAWI, A.H.; PIEGORSCH, W.W. (ed.). Encyclopedia of environmetrics. Chichester: John Wiley, 2002, v. 2. p. 1135-1142.

MCGARIGAL, K.; CUSHMAN, S. A. Comparative evaluation of experimental approaches to the study of habitat fragmentation effects. Ecological Applications, v. 12, n. 2, 2002, p. 335-345, 2002.

MCGARIGAL, K.; MARKS, B. J. Spatial pattern analysis program for quantifying landscape structure. Gen. Tech. Rep. PNW-GTR-351. US Department of Agriculture, Forest Service, Pacific Northwest Research Station, p. 1-122, 1995.

METZGER, J. P. O que é ecologia de paisagens? Biota Neotropica, São Paulo, v. 1, n. $1 / 2$, p. 1-9, 2001.

MYERS, N. et al. Biodiversity hotspots for conservation priorities. Nature, v.403, p. $853-858,2000$.

PEREIRA, J. L. G. et al. Métricas da paisagem na caracterização da evolução da ocupação da Amazônia. Geografia, Rio Claro, v. 26, n. 1, p. 59-90, 2001.

PÉRICO, E.; CEMIN, G. Planejamento do uso do solo em ambiente SIG: alocação de um distrito industrial no município de Lajeado, RS, Brasil. Estudos Geográficos, Rio Claro, v. 4, n. 1, p. 41-52, 2006.

PIROVANI, D. B.; GOMES DA SILVA, A.; ROSA DOS SANTOS, A. Análise da paisagem e mudanças no uso da terra no entorno da RPPN Cafundó, ES. Cerne [online], v. 21, n. 1, 2015.

POLETTO, M. C.; METZGER, J. P. A ecologia da paisagem na avaliação de impactos ecológicos de corredores rodoviários - o caso de um segmento do trecho sul do rodoanel de São Paulo. In: CONFERÊNCIA DA REDE DE LÍNGUA PORTUGUESA DE AVALIAÇÃO DE IMPACTOS; . $1^{\circ}$ CONGRESSO BRASILEIRO DE AVALIAÇÃO DE IMPACTO, 2., 1., 2012, São Paulo. Anais [...]. São Paulo: ABAI, 2012. p. 1-13.

REMPEL, C. A ecologia de paisagem e suas ferramentas podem aprimorar o zoneamento ambiental? $\mathrm{O}$ caso da região política do Vale do Taquari. 2009. 146 f. Tese (Doutorado em Ecologia) - Programa de Pós-Graduação em Ecologia, Universidade Federal do Rio Grande do Sul, Porto Alegre, 2009. 
RIBEIRO, S.; MARQUES, J. C. B. Características da paisagem e sua relação concorrência de bugios-ruivos (Alouatta guariba clamitans Cabrera, 1940; Primates Atelidae) em fragmentos florestais no vale do Taquari, RS. Natureza e Conservação, Amsterdam, v. 3, n. 2, p. 65-78, 2005.

SOARES, B. E. C. Desenvolvimento Sustentável e biodiversidade. Revista Biotecnologia Ciência \& Desenvolvimento, São Paulo, v. 33, p. 73-92, 2004.

SOUZA, C. G. et al. Análise da fragmentação florestal da área de proteção ambiental Coqueiral, Coqueiral-MG. Ciência Florestal [online], v. 24, n. 3, p. 631-644, 2014.

TABARELLI, M.; LOPES, A. V.; PERES, C. A. Edge effects drive tropical forest fragments towards an early successional system. Biotropica, New Jersey, v. 40, n. 6, p. 657-661, 2008.

TURNER, M. G. et al. Landscape ecology in theory and practice. New York: Springer, 2001.

UUEMAA, E.; MANDER, U.; MARJA, R. Trends in the use of landscape spatial metrics as landscape indicators: a review. Ecological Indicators [online], v. 28, p. 100-106, 2013.

VARJABEDIAN, R.; MECHI, A. As APPs de topo de morro e a Lei 12.651/12. In: CONGRESSO BRASILEIRO DE GEOLOGIA DE ENGENHARIA E AMBIENTAL, 14., 2013, Rio de Janeiro. Anais [...]. Rio de Janeiro: CPRM, 2013. p. 1-10. 
